Portland State University

PDXScholar

$1-1-2012$

\title{
In Vivo Quantification of Reactive Oxygen Species Demonstrates High Levels of Oxidative Stress in Base Excision Repair-Deficient Caenorhabditis Elegans: Implications for Associative Metabolic Phenotypes
}

Travis Lee Hase

Portland State University

Follow this and additional works at: https://pdxscholar.library.pdx.edu/honorstheses

Part of the Biology Commons

Let us know how access to this document benefits you.

\section{Recommended Citation}

Hase, Travis Lee, "In Vivo Quantification of Reactive Oxygen Species Demonstrates High Levels of Oxidative Stress in Base Excision Repair-Deficient Caenorhabditis Elegans: Implications for Associative Metabolic Phenotypes" (2012). University Honors Theses. Paper 10.

https://doi.org/10.15760/honors.10

This Thesis is brought to you for free and open access. It has been accepted for inclusion in University Honors Theses by an authorized administrator of PDXScholar. Please contact us if we can make this document more accessible: pdxscholar@pdx.edu. 
In vivo quantification of reactive oxygen species demonstrates high levels of oxidative stress in base excision repair8deficient Caenorhabditis elegans: implications for associative metabolic phenotypes

by

Travis Lee Hase

An undergraduate honors thesis submitted in partial fulfillment of the requirements for the degree of

Bachelor of Science

in

University Honors

and

Micro and Molecular Biology

Thesis Adviser

Suzanne R. Estes, Ph.D.

Portland State University 


\section{In vivo quantification of reactive oxygen species demonstrates high levels of oxidative stress in base excision repair-deficient Caenorhabditis elegans: implications for associative metabolic phenotypes}

\section{Introduction:}

Many factors and forces, both extrinsic and intrinsic, contribute to spontaneous mutation accumulation in all forms of life. As mutation is a common and expected occurrence, it becomes important to recognize and repair damaged DNA. Consequently, multiple DNA repair pathways have evolved to deal with the challenge that the diversity of mutagens presents to genomes. The various recognition and repair processes are one of the most important factors in minimizing mutation accumulation and propagation (Denver et al., 2006). Reactive oxygen species (ROS), such as the superoxide radial, hydroxyl radial, and $\mathrm{H}_{2} \mathrm{O}_{2}$ are the main contributors to stochastic, endogenous DNA damage (Ames et al., 1995). In addition to damaging DNA, oxidative stress from ROS also damages proteins, cellular lipids, and many other macromolecules (Sampath et al., 2011). Recent studies indicate that chronic oxidative stress greatly accelerates the development of obesity and other phenotypes associated with metabolic syndrome (see Discussion). Additionally, oxidative stress is implicated in aging and the development of agerelated diseases and degeneration (Ames et al, 1995 \& Finkel and Holbrook, 2000).

There are many sources of ROS, including mitochondria, which are capable of generating significant amounts (Starkov, 2008). Studies have shown that ROS are also generated outside the mitochondria, but that these sources are incapable of substantial ROS production, usually as a result of temporal occurrence or the availability of substrates (Droge, 2002). Finally, generation of ROS may also occur as 
a result of exposure to many exogenous sources, such as ionizing and ultraviolet radiation, environmental toxins, and inflammatory responses (Salmon et al., 2004). While ROS mediate a necessary role in signal transduction, the consequences of ROS exposure, albeit unavoidable, are generally considered to be adverse and biologically deleterious (Salmon et al., 2004 \& Starkov, 2008). Although ROS from all sources are capable of damaging DNA and other molecules, intramitochondrial ROS are of particular interest due to their high, continuous output.

Mitochondria are ubiquitously present in all nucleated cells and, unlike other cellular and exogenous sources, generate ROS continuously, most notably as a result of ATP production by oxidative phosphorylation. By means of the ETC, the mitochondrial inner membrane links oxidative phosphorylation to the energyreleasing activities of electron transport and proton pumping; however, this process is somewhat leaky and is subject to side reactions with molecular oxygen (Cadenas and Davies, 2000). Electrons which leak from the ETC react with molecular oxygen to form the superoxide radical, which can dismutate to form hydrogen peroxide and then further react to form the hydroxyl radical (Forman \& Boveris, 1982). The outer mitochondrial membrane is a significant source of hydrogen peroxide generation as a result of monoamine oxidase activity (Hauptmann et al, 1996). The majority of endogenous ROS result from leakage of these species from the inner and outer mitochondrial membranes (Finkel \& Holbrook, 2000). Thus, the steady state production of ROS via these toxic side reactions is a major cause of the adverse effects from oxidative stress, especially oxidative DNA and mtDNA damage. 
In eukaryotic cells, the three main DNA repair processes for single-stranded damage are base excision repair (BER), nucleotide excision repair (NER), and mismatch repair (MMR). The proper functioning of these different repair processes are central to maintaining genomic stability and preventing mutation accumulation. The nematode Caenorhabditis elegans prevents mutation accumulation through employment of all three of these processes (Denver et al., 2006). BER is widely believed to be the preferred mechanism for repair of oxidative DNA damage (Fensgård et al., 2010, Salmon et al., 2004, \& Sampath et al., 2011). Oxidative damage, as a result of ROS, commonly results in single and double strand breaks, DNA base modifications, apurinic lesions, and apyrimidinic lesions (Girard \& Boiteux, 1997). BER involves repairing the location of the base lesion or abasic site, DNA modifications resulting in minimal DNA helix distortion (Lindahl \& Wood, 1999). Whereas BER repairs lesions of minimal helix distortion, NER is predominantly responsible for correcting more bulky, helix-distorting lesions, commonly resulting from exposure to ultraviolet radiation (Salmon et al., 2004). In this study, I will focus upon deficiencies in BER with respect to endogenous ROS exposure.

In BER, DNA-glycosylases catalyze the first step of the repair process, in which the damaged base is removed from the helix, leaving the sugar-phosphate backbone intact while creating an AP site (Evert et al., 2004 \& Korkan et al., 2000). DNA glycosylases are ubiquitous across all kingdoms. Mammals, for example, possess 12 different DNA-glyscosylases and have much overlapping specificity. Due to the complexity of these repair systems and the presence of multiple 
compensatory responses, it becomes desirable to use a model system in which the consequences of BER-deficiency can be easily studied (Barnes \& Lindahl, 2004). C. elegans present themselves as a useful model in this study and others, as they encode only two DNA-glycosylases, NTH-1 and UNG-1 (Denver et al., 2006; Morinaga et al., 2009; Nakamura et al., 2008; Shatilla \& Ramotar, 2002). Due to the presence of alternate repair processes, overlapping specificities, and compensatory transcriptional responses, BER-deficient nematodes generally exhibit very mild phenotypes (Fensgård et al., 2010). Studies with Saccharomyces cerevisiae have linked DNA damage to the production of intracellular ROS (Salmon et al., 2004 \& Evert et al., 2004). In cells deficient in excision repair pathways, considerably high levels of spontaneous DNA damage accumulate with respect to the baseline controls with normal repair processes. Intracellular levels of ROS increase in a dose dependent manner with respect to increasing amounts of DNA damage (Evert et al., 2004). This correlation provides insight to a number of different disease states and implicates ROS as the etiologic agent, further demonstrating the deleterious effect of oxidative stress.

mtDNA is particularly vulnerable to damage from ROS, as it is near the site of production of ROS associated with the ETC, as well as it being without the protective features associated with gDNA such as NER and histones (Dingley et al., 2010). Although mtDNA is characterized by a higher level of mutagenesis (approximately a 10- to 15-fold increase) with respect to gDNA, when exposed to exogenous sources of oxidative stress, rather than endogenous, stochastic sources, mtDNA displays further increases in its mutation rate with respect to its baseline (Cadenas and 
Davies, 2000 \& Sampath et al., 2011). A high rate of irreparable mutation affects a mitochondrion's ability to produce ATP and otherwise function properly, distorting energy homeostasis. It is clear that structural, metabolic, and genetic changes within organelles can contribute to whole-body physiology. Decreased mitochondrial capacity alone contributes to a number of different conditions, many of them metabolically related (Salmon et al., 2004 \& Mantena et al., 2008).

Metabolic syndrome describes a number of interrelated disorders largely comprised of obesity, dyslipidemia, type-two diabetes mellitus, and hypertension, which result in atherosclerosis and coronary artery disease (Moller \& Kaufman, 2005). Do their prevalence, the diseases associated with metabolic syndrome currently pose a threat to public health, and there is much interest in understanding the underlying biology behind disorders associated with aging, mutagenesis, and organellar function.

In this present study, I use C. elegans as a model organism to better characterize the phenotypic response to deficiencies in BER. This will help to elucidate how DNA damage, increased mutagenesis, and exposure to ROS is related to Metabolic Syndrome. Additionally, this work will establish a starting point for investigation that can further characterize phenotypic responses such as mitochondrial physiology and morphology through measurements of mitochondrial membrane potential and mitochondrial circularity. The overall goal of this research is to better understand the nature of mitochondrial dysfunction with respect to the inactivation of DNA glycosylases, as well as understand the contribution DNA repair processes have to the maintenance of energy homeostasis and substrate oxidation. 


\section{Materials \& Methods:}

\section{Nematode Strains and Culture Conditions}

For this current study, all four C. elegans nematode strains were kindly provided by The Biotechnology Centre (University of Oslo, Blindern, Oslo, Norway). Strains consisted of an N2 wildtype ( $\mathrm{nth}^{+/+}$; $\left.\mathrm{ung}^{+/+}\right)$; two single DNA glycosylase

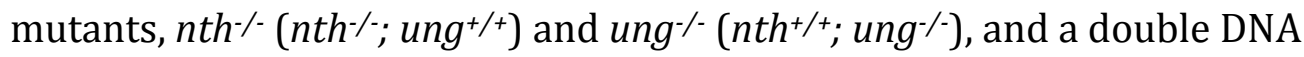
glycosylase mutant ( $\mathrm{th}^{-/}$; ung ${ }^{-/}$). As C. elegans encode only two DNA glycosylases, we are able to subsequently remove all BER activity with the double knockout strain.

All nematode strains were maintained under standard laboratory conditions at $20^{\circ} \mathrm{C}$ on $15 \mathrm{~mm}$ NGM Petri plates treated with Streptomycin and seeded with Escherichia coli str. OP50-1 as a food source. All strains were included in each analysis. Prior to each assay, age synchronous worms were obtained by means of a standard bleaching protocol.

\section{Fluorescence Confocal Microscopy}

We performed confocal image analysis on young adult nematodes treated with a mitochondria-specific fluorescent dye. Fluorescent imaging allows for simultaneous localization and relative quantification of mitochondrial traits. Additionally, recent work indicates that ROS data obtained from fluorescent analysis and electron spin resonance yield similar results (Kuznetsov et al., 2011). Prior to each assay, age synchronous worms were incubated with E. coli str. OP50-1 and $10 \mu \mathrm{M}$ of MitoSOX Red (Invitrogen). Concurrently, age synchronous worms of the 
same strains were incubated without dye to serve as controls. After 24 hours of incubation, young adult stage worms were washed and transferred to fresh NGM plates containing unlabeled E. coli str. OP50-1. The worms were allowed to feed for one hour, cleaning their digestive tracts of any labeled E. coli, which would interfere with accurate fluorescence measurements. Immediately prior to imaging, the worms were paralyzed with one drop of 5M levamisole (Molecular Probes, Inc., Eugene, OR, Carlsbad, CA) following Dingley et al. (2009). Levamisole paralyzes worms by preventing depolarization of skeletal muscles (Lewis et al., 1980).

For each assay, images of the pharynx of each nematode were collected. The pharynx is a neuromuscular organ that nematodes use for feeding (Albertson \& Thomson, 1976). The pharynx was chosen for these assays not only because it is a mitochondria-rich tissue, but also because, as a neuromuscular organ, it is strikingly similar to the mammalian heart (Salkoff et al., 2005 \& Mango, 2007). Additionally, the pharyngeal bulb is easily visualized and has high levels of dye uptake. As the pharynx contains few lipid droplets, there is little interference with accurate measurement of mitochondrial fluorescence, as lipids are highly autofluorescent.

Images were acquired using a high resolution, wide field Core DV system (Applied Precision $^{\mathrm{TM}}$, Issaquah, WA) equipped with an Olympus IX71 inverted microscope mounted with a Nikon Coolsnap ES2 HQ camera (Advanced Light Microscopy Core Facility, Oregon Health and Science University, Portland, OR). Fluorescent z-stack images of the pharyngeal bulb of individual worms were captured at 60X magnification using a short arc 250W Xenon lamp. Images of the 
pharynx were captured with a 1.0s exposure time. All images were acquired immediately following paralysis of the worms.

\section{Relative Mitochondrial Oxidant Levels}

ROS levels were measured in vivo for all C. elegans strains using the basic approach of Dingley et al. (2009) and described further in Estes et al. (2011) and Hicks et al. (2012 Submitted). In brief, worms were washed off of NGM plates with M9 buffer, gently centrifuged into a pellet, and then transferred onto fresh NGM plates seeded with $E$. coli str. OP50-1, which had been incubated with $10 \mu \mathrm{M}$ MitoSOX Red (Molecular Probes, Inc., Eugene, OR). At this point, all samples were shielded from light due to the light sensitivity of the dye. Under these conditions, worms were incubated for 24 hours prior to imaging, as outlined above.

MitoSOX Red is a mitochondrial-specific, fluorogenic dye. It is preferentially taken up by respiring mitochondria and is oxidized by superoxide, allowing for the quantification of ROS levels. Additionally, MitoSOX Red was recently shown to quantify total levels of mitochondrial oxidants, rather than superoxide specifically, when used with confocal microscopy (Zielonka \& Kalyanaraman, 2010).

\section{Image Analysis}

All image analyses were preformed using ImageJ software (National Institutes of Health, Bethesda, MD, v. 1.43u). The z-stack images were first optimized by deconvolving. Terminal pharyngeal bulbs were then manually encircled by use of the "eclipse" function within each image to quantity mean 
fluorescence intensity of the area. This was done for both exposed and unexposed (control) worms for each strain. The final intensity of the bulb is calculated as the difference between intensity values for exposed and unexposed worms.

Fluorescence increases in a dose-dependent manner with respect to increasing ROS, such that relative fluorescence values allow for the quantification of relative ROS.

Mitochondrial Localization of Fluorescent Dyes and Effect of Levamisole

Previous work by the Estes Lab (Department of Biology, Portland State University, Portland, OR) has studied the effect of levamisole on dye fluorescence (data not shown). Regarding MitoSOX Red, there was no effect on the maximum values of fluorescence; however, there was a significant effect of levamisole on mean MitoSOX Red fluorescence (Hicks, 2012 Submitted). Therefore, maximum fluorescence values were used for all statistical analyses, such that any strain-byprobe interactions generated by levamisole were unlikely to influence the amongstrain comparisons. Additionally, maximum measurements are more consistent in fluorescence image analyses, as they are unaffected by variation in pixel size and mitochondrial number.

\section{Results:}

Upon feeding mitochondria-specific, fluorescent MitoSOX Red dye to C. elegans, a consistent pattern of preferential uptake was observed within the pharyngeal bulb in the N2, ung $/$, and $n t h / / u^{-/}$strains (see Figure 1). This labeling was not consistently observed in the $n t h \%$ worms, indicating a lack of uptake. 

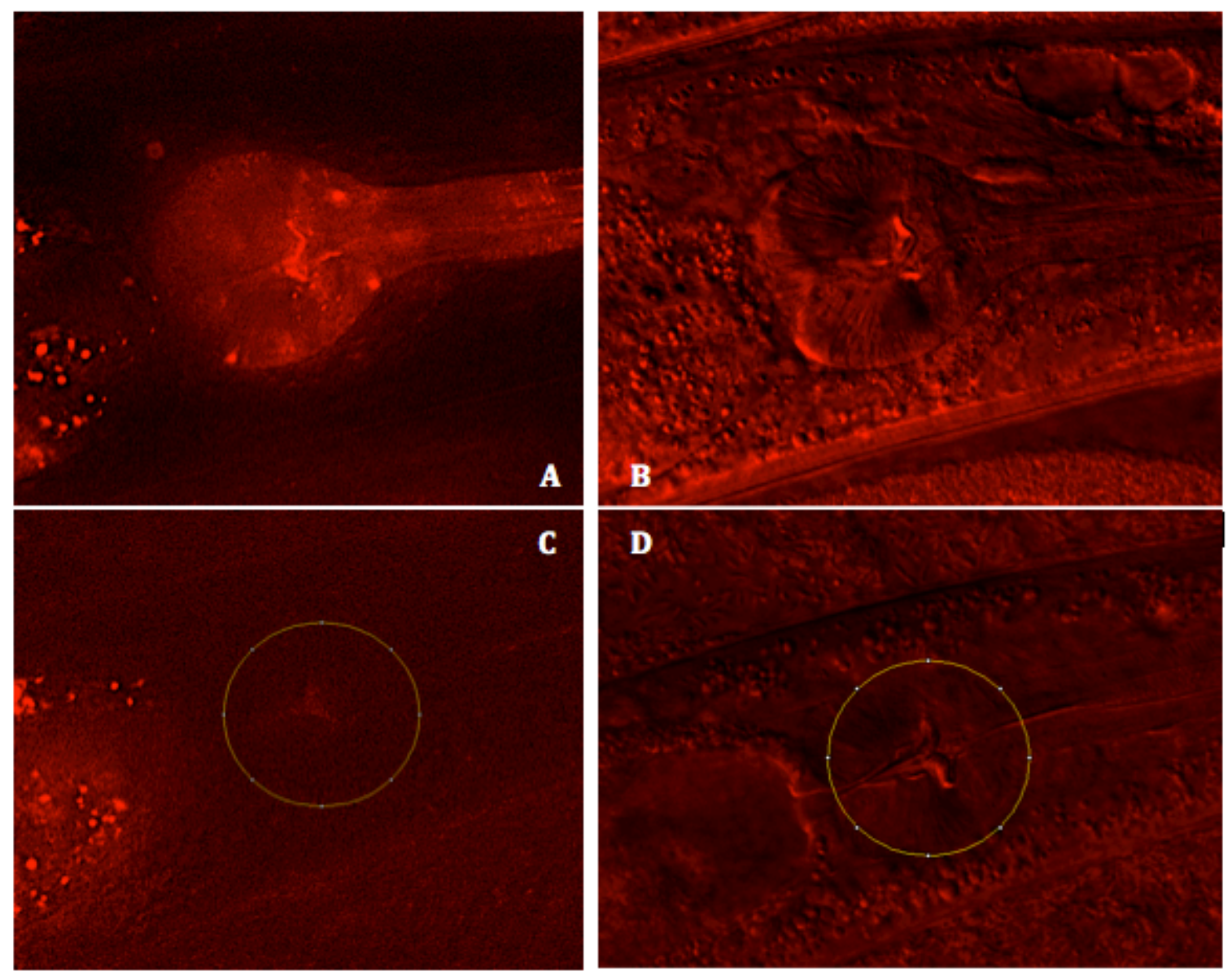

Figure 1: Nematode terminal pharyngeal bulb labeling with mitochondria-targeted MitoSOX Red florescent dye. (A) Singe z-stack micrograph of a young adult ung $\%$ C. elegans nematode exposed to MitoSOX Red, demonstrating consistent labeling within the pharynx, as well as in the lipid-rich gut granules that non-specifically bind lipophilic dyes. (B) Reference image of the same nematode as described in (A), demonstrating the nematode anatomy as viewed with confocal microscopy (60X). (C) Singe z-stack micrograph of a young adult ung $\%$ C. elegans nematode unexposed to MitoSOX Red (control), demonstrating the absence of a labeled pharynx (as outlined), as well as the autoflorescence of the lipid-rich gut granules. (D) Reference image of the same nematode as described in (C), demonstrating the nematode anatomy as viewed with confocal microscopy (60X).

\section{Relative fluorescence was calculated by subtracting the mean maximum}

fluorescence values of 5 non-dyed worms of each strain from the mean maximum

$$
\text { fluorescence values of } 20 \text { dyed worms of each strain (see Table 1). }
$$




\begin{tabular}{c|cccc}
\hline Fluorescence & N2 & nth $/-$ & ung $^{\%}$ & nth $^{-/}$; ung $^{-/-}$ \\
\hline Control & 405.61 & 589.84 & 351.19 & 295.45 \\
MitoSOX & 1502.95 & 379.42 & 1107.27 & 913.31 \\
Relative & 1097.34 & -210.42 & 756.08 & 617.86 \\
\hline
\end{tabular}

Table 1: Relative mean fluorescence calculations from maximum fluorescence measurements in all four strains.

As reported in the literature (see Discussion below), it should be observed that worms with mutations in DNA repair processes should have elevated ROS, as measured by the amount of fluorescence, with respect to the wildtype. Therefore, the results reported here are inconsistent with expectations. It is worth addressing the potential sources of these inconsistencies, of which there are many. One of the most obvious is exposure to light. As the dyes are light sensitive, it is of the highest importance that a dark atmosphere be maintained during the dying and incubation processes. Failure to prevent exposure to light leads to photobleaching and will negatively affect fluorescence levels. Additionally, ROS levels may be sufficiently high in the mutants, such that quenching is occurring. Therefore, it would be useful to alter dye exposure times and dye concentrations. Although age synchronous, factors such as worm development are also subject to variance and may have an affect. Lastly, there are a number of steps prone to standard experimental error. Regardless, the results obtained here do not match widely reported observations, and it is certain that a repeat of the staining and imaging analysis needs to be undertaken. For this reason, the Discussion will proceed as if it was observed that a statistically significant effect was observed such that the wildtype had the lowest level of ROS, the single mutants had midlevel amounts of ROS, and the double mutant demonstrated the highest level of ROS. Moving forward with this 
assumption is consistent with the literature, and it will serve as the basis of the discussion until further experimentation refines the data.

\section{Discussion \& Outlook:}

Studies by Mantena et al. (2008), Taylor and Turnbull (2005), and Tuppen et al. (2001) in both human- and animal-based models, have shown a relationship between the loss of DNA glycolase activity and a predisposition to obesity, indicating a link between DNA repair mechanisms, DNA damage, and metabolic syndrome (Sampath et al., 2011). Additionally, similar reductions in mtDNA have been described in other metabolic conditions. For example, mitochondriallygenerated NAD is critical to fatty acid oxidation. Given that oxidative stress will result in mitochondrial insufficiencies, it is consistent that increased adiposity is observed in animal models that lack DNA repair enzymes and, subsequently, display a phenotypically-altered ETC, such that NAD is not produced to the extent necessary for fat oxidation, leading to its accumulation in adipocytes (Sampath et al., 2011). Additionally, Sampath et al. (2011) have shown that deficiency in NEIL-1, a DNA glycosylase, increases the susceptibility to weight gain and obesity in neil-1\% mice. In addition to weight gain, the experimental model also shows a significant increase in plasma triglycerides and hepatic lipid accumulation. Mantena et al. (2008) have shown strong connections between mitochondrial dysfunction—largely an inability to produce sufficient amounts of ATP-and oxidative stress in the pathogenesis of alcohol- and obesity/type-2 diabetes-induced fatty liver disease. Specifically, the inflammatory response associated with these diets increases the production of ROS 
and reactive nitrogen species, which subsequently damage the mitochondrial genome. Damage to mtDNA leads to alterations in the mitochondrial proteome and a loss off ability for mitochondrial respiration to maintain sufficient ATP concentrations. In a cyclic fashion, this mitochondrial dysfunction leads to even more oxidative stress (Mantena et al, 2008).

Indeed, the role of oxidative stress in the disease processes associated with metabolic syndrome have been demonstrated and well characterized in a number of different experimental models. It is known that increased levels of ROS are indicative of damaged DNA , and it has been well documented that cells deficient in DNA repair processes accumulate mutations through spontaneous damage to the genome (Salmon et al., 2004 \& Evert et al., 2004).

The findings of this study certainly indicate the presence of spontaneous DNA and mtDNA damage that remains unrepaired. Logic would follow that this unrepaired damage has resulted in changes to the mitochondria that affect their ability to function and maintain energy homeostasis; however, further evaluation is required to support this assumption. Specifically, further studies of mitochondrial physiology and morphology should include measurements of mitochondrial membrane potential and mitochondrial circularity. Membrane potential will be measured using MitoTracker Red CMXRos (Invitrogen). Uptake of this die is dependent upon membrane potential, such that increased potential is associated with greater uptake, and subsequently, greater amounts of fluorescence (Pendergrass et al., 2009). Circularity is measured using both MitoTracker Red CMXRos and MitoTracker Green FM (Invitrogen), concurrently. Unlike MitoTracker 
Red CMXRos, MitoTracker Green FM is not dependent on mitochondrial membrane potential. This will permit the labeling of both actively and non-actively respiring mitochondria. Differential labeling permits an analysis to determine if mitochondrial membrane potential correlates with changes in morphology. By documenting these easily quantifiable mitochondrial phenotypes, it will be easier to understand how the ability of the mitochondria to function is affected. Lastly, further investigation is required to identify and better understand the molecular mechanisms, as well as quantify and identify the damage these elevated ROS levels point to.

The susceptibility of mtDNA to damage from oxidative stress is well documented and has been ascribed to a number of factors. Additionally, it has been consistently observed that mitochondrial dysfunction can contribute to the wholebody pathologies associated with Metabolic Syndrome. Therefore, a goal for current and future investigations in the field will be to provide a characterization of the molecular processes involved in these pathologies. A more complete understanding of the oxidative stress-mediated effects on the spectrum of metabolic diseases is needed to facilitate the development of treatments. This work highlights the utility of C. elegans as a model that allows for the non-invasive, in vivo observation of the consequences of mitochondrial dysfunction. 


\section{References:}

Albertson, D.G. \& Thomson, J.N. (1976). The pharynx of Caenorhabditis elegans. Philosophical Transactions of the Royal Society B: Biological Sciences, 275, 299-325.

Ames, B.N., Shigenaga, M.K., \& Hagen, T.M. (1995). Mitochondrial decay in aging. Biochemica et Biophysica Acta, 1271, 15-170.

Barnes, D.E. \& Lindahl, T. (2004). Repair and genetic consequences of endogenous DNA base damage in mammalian cells. Annual Review of Genetics, 38, 445476.

Cadenas, E. \& Davies, K.J.A. (2000). Mitochondrial free radical generation, oxidative stress, and aging. Free Radical Biology and Medicine 29, 222-230.

Denver, D.R., Feinberg, S., Steding, C., Durbin, M., and Lynch, M. (2006). The Relative Roles of Three DNA Repair Pathways in Preventing Caenorhabditis elegans Mutation Accumulation. Genetics, 174, 57-65.

Dingley, S.E. et al. (2009). Mitochondrial respiratory chain dysfunction variably increases oxidant stress in Caenorhabditis elegans. Mitochondrion.

Droge, W. (2002). Free radicals in the physiological control of cell function. Physiological Reviews, 82, 47-95.

Estes, S., Coleman-Hulbert, A., Hicks, K.A., de Haan, G., Martha, S.R., Knapp, J.B., Smith, S.W., Stein, K.C., \& Denver, D.R. (2011). Natural variation in life history and aging phenotypes is associated with mitochondrial DNA deletion frequency in Caenorhabditis briggsae. BMC Evolutionary Biology, 11:11.

Evert, A., Salmon, T., Song, B., Jingjing, L., Siede, W., \& Doetsch, P. (2004). Spontaneous DNA Damage in Saccharomyces cerevisiae Elicits Phenotypic Properties Similar to Cancer Cells. The Journal of Biological Chemistry, 279(21), 22585-22594.

Fensgård, Ø., Kassahun, H., Bombik, I., Torbjorn, R., Lindvall, J., \& Nilsen, H. (2010). A Two-tiered compensatory response to loss of DNA repair meodulates aging and stress response pathway. Aging , 2(3), 133-159.

Finkel, T. \& Holbrook, N.J. (2000). Oxidants, oxidative stress and the biology of ageing. Nature, 408, 239-247.

Forman, H.J. \& Boveris, A. (1982). Superoxide radical and hydrogen peroxide in mitochondria. Free Radicals in Biology, 65-90. 
Girard, P.M. \& Boiteux, S. (1997). Repair of oxidized DNA bases in the yeast Saccharomyces cerevisiae. Biochimie, 79, 559-566.

Hauptmann, N., Grimsby, J., Shih, J., Shih, J.C., \& Cadenas, E. (1996). The metabolism of tyramine by monoamine oxidase A/B causes oxidative damage to mitochondrial DNA. Archives of Biochemistry and Biophysics, 335, 295-304.

Hicks, K.A., Howe, D., Leung, A., Denver, D., \& Estes, S. (2012 Submitted). In vivo quantification reveals extensive natural variation in mitochondrial form and function in Caenorhabditis briggsae. Evolution.

Krokan, H.E. et al. (2000). Base excision repair of DNA in mammalian cells. FEBS Letters, 476, 73-77.

Kuznetsov, A.V. et al. (2011). Mitochondrial ROS production under cellular stress: comparison of different detection methods. Analytical and Bioanalytical Chemistry, 400, 2383-2390.

Lewis, J.A., Wu, C.H., Levine, J.H., \& Berg, H. (1980). Levamisole-resistant mutants of the nematode Caenorhabditis elegans appear to lack pharmacological acetylcholine receptors. Neuroscience, 5, 967-989.

Lindahl, T. \& Wood, R.D. (1999). Quality control by DNA repair. Science, 286, 18971905.

Mango, S.E. (2007). The C. elegans pharynx: a model for organogenesis. WormBook, 1-26.

Mantena, S.K., King, A.L., Andringa, K.K., Eccleston, H.B., \& Bailey, S.M. (2008). Mitochondrial dysfunction and oxidative stress in the pathogenesis of alcohol- and obesity-induced fatty liver disease. Free Radical Biology and Medicine, 44, 1259-1272.

Meissner, B., Rogalski, T., Viveiros, R., Warner, A., Plastino, L., Lorch, A., et al. (2011). Determing the Sub-Cellular Localization of Proteins within Caenorhabditis elegans Body Wall Muscle. PLoS ONE , 6(5), e19937 1-16.

Moller, D.E. \& Kaufman, K.D. (2005). Metabolic Syndrome: A Clinical and Molecular Perspective. Annual Review of Medicine, 56, 45-62.

Morinaga, H. et al. (2009). Purification and characterization of Caenorhabditis elegans NTH, a homolog of human endonuclease III: essential role of Nterminal region. DNA Repair, 8, 844-851. 
Nakamura, N. et al. (2008). Cloning and characterization of uracil-DNA glycosylase and the biological consequences of the loss of its function in the nematode Caenorhabditis elegans. Mutagenesis, 23, 401-413.

Pendergass, W., Wolf, N., \& Poot, M. (200\$) Efficacy of MitoTracker Green and CMXrosamine to measure changes in mitochondrial membrane potentials in living cells and tissues. Cytometry A, 61(2), 162-169.

Salkoff, L. et al. (2005). Potassium channels in C. elegans. WormBook, 1-15.

Salmon, T., Evert, A., Song, B., \& Doetsch, P. (2004). Biological consequences of oxidative stress-induced DNA damage in Saccharomyces cerevisiae. Nucleic Acids Research, 32(12), 3712-3723.

Sampath, H., Batra, A., Vartanian, V., Carmical, R., Prusak, D., King, I., et al. (2011). Variable penetrance of metabolic phenotypes and development of high-fat diet-induced adiposity in NEIL1-deficient mice. American Journal of Physiology, Endocrinology, and Metabolism, 300, E724-E734.

Shatilla, A., \& Ramotar, D. (2002). Embryonic extracts derived from the nematode Caenorhabditis elegans remove uracil from DNA by the sequential action of uracil-DNA glycosylase and AP (apurinic/apyrimidinic) endonuclease. Biochemical Journal, 365, 547-553.

Starkov, A.A. (2008). The Role of Mitochondria in Reactive Oxygen Species Metabolism and Signaling. Mitochondria and Oxidative Stress in Neurodegenerative Disorders, 1147, 37-52.

Taylor, R.W. \& Turnbull, D.M. (2005). Mitochondrial DNA mutations in human disease. Nature Reviews Genetics, 6, 389-402.

Tuppen, H.A., Blakely, E.L., Turnbull, D.M., \& Taylor, R.W. (2010). Mitochondrial DNA mutations and human disease. Biochimica et Biophysica Acta, 1797, 113-128.

Zielonka, J. \& Kalyanaraman, B. (2010). Hydroethindine- and MitoSOX-derived red fluorescence is not a reliable indicator of intracellular superoxide formation: another inconvenient truth. Free Radial Biology and Medicine, 48, 983-1001. 